\title{
A nutrition score based on public health indicators of the French Nutrition and Health Programme: relationships with nutritional status and the risk of major chronic diseases in the Supplementation en Vitamines et Mineraux Antioxydants (SU.VI.MAX) cohort
}

\author{
C. Estaquio ${ }^{1}$, K. Castetbon ${ }^{2}$, E. Kesse-Guyot ${ }^{1}$, S. Bertrais ${ }^{1}$, V. Deschamps ${ }^{2}$, L. Dauchet ${ }^{1}$, \\ S. Péneau ${ }^{1}$, P. Galan ${ }^{1}$ and S. Hercberg ${ }^{1}$ \\ ${ }^{1}$ UMR U557 INSERM/U1125 INRA/CNAM/Paris 13 Université/Centre de Recherche en Nutrition Humaine -IdF, \\ Bobigny, France and ${ }^{2}$ Unité de Surveillance et d'Epidémiologie Nutritionnelle, Institut de Veille Sanitaire (InVS)/ \\ Paris 13 Université/CNAM/Centre de Recherche en Nutrition Humaine -IdF, Bobigny, France
}

Few studies have found that adherence to the dietary guidelines reduces the incidence of chronic disease ${ }^{(1)}$. In 2001 a National Nutrition and Health Programme (Programme National Nutrition Santé; PNNS) was implemented in France, which included nine priority quantified nutritional goals ${ }^{(2)}$. The present study has developed an index score that includes these public health objectives and has examined associations between this score and the incidence of major chronic diseases in the SU.VI.MAX study.

The SU.VI.MAX study ${ }^{(3)}$ was initially designed as a randomized double-blind placebo-controlled primary-prevention trial to test the efficacy of daily supplementation with antioxidant vitamins and minerals at nutritional doses in reducing the incidence of IHD and cancer. Women were 35-60 years of age and men were 45-60 years of age at enrolment. Throughout the study respondents completed food records, health and demographic questionnaires, provided blood samples and underwent physical examinations.

The construction of the French score of indicators of the PNNS objectives is based on the identification of a 'healthy' threshold for each objective by using the statement of the objective itself. Scoring decisions were made a priori based on the PNNS objectives or on epidemiological or clinical evidence. The general approach was to attribute one point (maximum score) to subjects who reached the PNNS indicator threshold, with the exception of physical activity for which additional points were given to subjects who exceeded the current guidelines. For each component, intermediate points ( 0.5 points) were attributed to those who did not entirely reach the threshold but whose nutritional status was considered 'fair'. The remaining subjects did not receive any points. A priori weighting for each index component were also included. Moreover, points were deducted for individuals with energy intakes greater than their estimated needs.

Survival analyses were performed by gender. The first 2 years of the follow-up (1994-5) were excluded in order to eliminate subjects who may had a pre-existing disease. So, follow-up time was the time between the end of the first 2 years of follow-up (1996-7) and the first event (CVD, cancer, death or last contact) or until the day the subject completed 10 years of follow-up (2004-5). For survival analyses a major chronic disease was defined as the combination of CVD, cancer or death, whichever came first. Data from middle-aged adults who were free of major chronic diseases and who provided at least three $24 \mathrm{~h}$ dietary records during the first 2 years of follow-up were included in the present analyses ( $n$ 4976).

Major chronic disease documented during the 8-year follow-up period ( $n$ 455) was defined as the combination of CVD ( $n$ 131), cancer ( $n$ 261) or death ( $n$ 63), whichever came first. In fully-adjusted Cox models men in the highest score tertile compared with those in the lowest score tertile had a $36 \%$ lower risk of major chronic disease (hazard ratio 0.64 (95\% CI 0.43, 0.94)). No association was found in women.

These results demonstrate the potential for improving the health status of the French population, particularly in men, if the PNNS nutrition guidelines are followed by changes in habits. They also support interest in pursuing a public health nutrition programme such as the PNNS.

1. Waijers PM, Feskens EJ \& Ocke MC (2007) Br J Nutr 97, 219-231.

2. Ministère de la Santé (2007) Programme National Nutrition Santé (2001-2005). http://www.sante.gouv.fr/htm/pointsur/nutrition/1n1.pdf (accessed December 2007).

3. Hercberg S, Galan P, Preziosi P, Bertrais S, Mennen L, Malvy D, Roussel AM, Favier A \& Briançon S (2004) Arch Intern Med 164, $2335-2342$. 